\title{
Optimality Conditions for Solutions to Hyperbolic Balance Laws
}

\author{
Alberto Bressan and Wen Shen \\ Department of Mathematics, Penn State University \\ University Park, Pa. 16802, U.S.A. \\ e-mails: bressan@math.psu.edu, shen_w@math.psu.edu
}

\begin{abstract}
We study a class of optimization problems for solutions to a hyperbolic system of balance laws. The controller can affect the evolution of the system through the source terms, as well as the boundary conditions. Our main goal is to obtain necessary conditions in order that a piecewise regular solution, with finitely many shocks, be optimal. These necessary conditions are derived by constructing a family of "generalized cotangent vectors", which can be transported backwards along a trajectory.
\end{abstract}

\section{1 - Introduction}

Aim of this paper is to derive necessary conditions for the optimality of solutions to a hyperbolic system of balance laws, with boundary conditions. Consider a one-dimensional system of conservation laws with source terms

$$
u_{t}+[F(u)]_{x}=g(x, u, w), \quad t \in[0, T], \quad x \geq 0 .
$$

Here $u \in \mathbb{R}^{n}$ is the vector of conserved quantities, while $f: \mathbb{R}^{n} \mapsto \mathbb{R}^{n}$ is the flux function. The source term $g$ also depends on a control variable $w=w(t)$, taking values inside a convex set $W \subset \mathbb{R}^{m}$. The above system will be supplemented by the initial condition

$$
u(0, x)=\bar{u}(x)
$$


together with boundary conditions. Throughout the following, we assume that the system is strictly hyperbolic, so that each Jacobian matrix $A(u) \doteq D F(u)$ has $n$ real distinct eigenvalues. To fix the ideas, assume

$$
\lambda_{1}(u)<\cdots<\lambda_{p}(u)<0<\lambda_{p+1}(u)<\cdots<\lambda_{n}(u) .
$$

This implies that at the boundary point $x=0$ there are $n-p$ characteristics entering the domain. We then impose $n-p$ boundary conditions of the form

$$
\phi_{j}(u(t, 0+), z(t))=0 \quad j=1, \ldots, n-p .
$$

Here $t \mapsto z(t)$ denotes a boundary control function, taking values in a convex set $Z \subseteq \mathbb{R}^{m^{\prime}}$. We shall assume that the above boundary conditions are linearly independent and, for given control functions $w, z$, the solution of the initial-boundary value problem (1.1)-(1.3) is well defined [AC].

Among all solutions to the above problem, we wish to select one that maximizes a certain payoff. This may depend on:

- the values of the solution $u$ and of the control $w$ on the entire domain $[0, T] \times[0, \infty[$,

- the profile of the solution $u$ at the terminal time $t=T$,

- the values of the solution $u$ and of the control function $z$ along the boundary $x=0$.

For sake of generality, we thus consider a payoff of the form

$$
J(u, w, z) \doteq \int_{0}^{T} \int_{0}^{\infty} L(x, u, w) d x d t+\int_{0}^{\infty} \varphi(x, u(T, x)) d x+\int_{0}^{T} \psi(u(t, 0+), z(t)) d t .
$$

The optimization problem will thus take the form

$$
\max _{w \in \mathcal{W}, z \in \mathcal{Z}} J(u, w, z)
$$

Here $\mathcal{W}$ is the family of all measurable control functions $w=w(t, x)$ taking values inside the set $W \subset \mathbb{R}^{m}$, while $\mathcal{Z}$ is the set of admissible boundary control functions $z:[0, T] \mapsto Z$. Of course, $u=u(t, x)$ denotes the solution of the initial-boundary problem (1.1)-(1.3) corresponding to the control functions $w, z$.

We seek necessary conditions, in order that the couple of control functions $\hat{w}=\hat{w}(t)$ and $\hat{z}=\hat{z}(t)$ be optimal. To derive such conditions, one needs to understand how the values $u(t, x)$ of the solution of (1.1)-(1.3) are affected, if the controls $w, z$ are varied.

Compared with more standard optimization problems, the main difficulty faced in the present setting is that the "input to trajectory" map $(w, z) \mapsto u$ may not be differentiable in any natural Banach space. Indeed, consider a family of control functions $\left\{\left(w^{\theta}, z^{\theta}\right) ; \theta \geq 0\right\}$ smoothly depending on the scalar parameter $\theta$. Assume that, at a given time $t$, the corresponding solutions $u^{\theta}(t, \cdot)$ contain a shock, whose location $x^{\theta}(t)$ varies with $\theta$. Then the derivative

$$
\lim _{h \rightarrow 0} \frac{u^{\theta+h}(t)-u^{\theta}(t)}{h}
$$

cannot be represented by a function. Indeed, the limit (1.6) is not well defined in any function space. It can only be interpreted as a vector measure, containing a point mass at $x=x^{\theta}(t)$. 
Assuming that the reference solution $\hat{u}$ is piecewise smooth with finitely many (possibly interacting) shocks, the behavior of a slightly perturbed solution $u^{\varepsilon}$ can be described in terms of "shift differentials", as introduced in [BM1]. In the subsequent paper [BM2], the authors introduced a class of "generalized cotangent vectors" and derived an adjoint system of linear equations and boundary conditions, determining how these covectors are transported backward in time along the trajectory $\hat{u}$. This yielded a necessary condition for the optimality of a (sufficiently regular) control $\hat{w}$.

The results in [BM2] dealt with solutions defined on the whole real line, and with a payoff functional depending only on the terminal value $u(T, \cdot)$. In the present paper, after reviewing the theory of generalized differentials, we derive new optimality conditions which cover the more general case where

- solutions $u$ are defined on a half line, and the control affects the boundary conditions at $x=0$,

- shock interactions can occur within the optimal solution,

- the payoff includes a term depending on boundary values.

An even more general setting would be provided by equations of the form

$$
u_{t}+[F(u)]_{x}=g(t, x, u, w(t, x)), \quad w(t, x) \in W \subset \mathbb{R}^{m},
$$

where the control $w$ is a function of both variables space and time. For notational simplicity we shall restrict our study to the system (1.1). However, our analysis can be adapted without much difficulty to this more general case, since the linearized equations used to transport tangent and cotangent vectors along a given trajectory are exactly the same.

As in [BM2], the main technical problem faced in the proof is that the transport equations for tangent vectors can be justified only under the a-priori assumption that all perturbed solutions $u^{\varepsilon}$ remain piecewise Lipschitz continuous, with the same number of jumps as $\hat{u}$. Therefore, when a family $\left\{\left(w^{\varepsilon}, z^{\varepsilon}\right)\right\}$ of control variations is constructed, one has to check that the corresponding solutions $u^{\varepsilon}=u\left(z^{\varepsilon}\right)$ do not develop a gradient catastrophe before the terminal time $T$. For this reason, we shall require strong regularity assumptions on the optimal control $(\hat{w}, \hat{z})$ and on the optimal solution $\hat{u}$. Our main theorem provides necessary conditions for the optimality of a solution to the initial-boundary value problem (1.1)-(1.3).

We remark that here all functions $F, g, \phi_{j}, L, \varphi, \psi$, determining the evolution equation and the payoff functional, are assumed to be smooth but can be strongly nonlinear. In the special case where the payoff is given by a linear functional, it becomes possible to consider first order variations within the larger space of measures. This leads to a different approach to optimality conditions, for which we refer to [BJ].

\section{2 - Basic assumptions and notations.}

In the following, $|\cdot|$ and $\langle\cdot, \cdot\rangle$ denote the Euclidean norm and inner product on $\mathbb{R}^{m}$, respectively. We always assume that the following conditions hold:

(H1) The flow function $F: \Omega \mapsto \mathbb{R}^{n}$ in (1.1) is smooth on a domain $\Omega \subseteq \mathbb{R}^{n}$. The system (1.1) is strictly hyperbolic, with each characteristic field either linearly degenerate or genuinely nonlinear. The source term $g$ is a smooth function of $x, u, w$. 
We denote by $\lambda_{i}(u), r_{i}(u), l_{i}(u)$ respectively the $i$-th eigenvalue and $i$-th right and left eigenvector of the Jacobian matrix $A(u)=D F(u)$, normalized so that

$$
\left|r_{i}(u)\right| \equiv 1, \quad\left\langle l_{i}(u), r_{j}(u)\right\rangle \equiv \delta_{i j}= \begin{cases}1 & \text { if } i=j, \\ 0 & \text { if } i \neq j .\end{cases}
$$

For $u, u^{\prime} \in \mathbb{R}^{n}$, define the averaged matrix

$$
A\left(u, u^{\prime}\right)=\int_{0}^{1} A\left(\theta u+(1-\theta) u^{\prime}\right) d \theta .
$$

Clearly $A\left(u, u^{\prime}\right)=A\left(u^{\prime}, u\right)$ and $A(u, u)=A(u)$. For $i=1, \ldots, n$, the $i$-th eigenvalue and eigenvectors of $A\left(u, u^{\prime}\right)$ will be denoted by $\lambda_{i}\left(u, u^{\prime}\right), r_{i}\left(u, u^{\prime}\right), l_{i}\left(u, u^{\prime}\right)$. We assume that the ranges of the eigenvalues $\lambda_{i}$ do not overlap, i.e. that there exist disjoint intervals $\left[a_{i}, b_{i}\right]$ such that

$$
\lambda_{i}\left(u, u^{\prime}\right) \in\left[a_{i}, b_{i}\right] \quad \forall u, u^{\prime} \in \Omega, i \in\{1, \ldots, m\} .
$$

Moreover, we assume that

$$
b_{p}<0<a_{p+1},
$$

so that at the boundary of the domain $\{x \geq 0\}$ there will always be $p$ exiting and $n-p$ entering characteristics. If $\phi$ is any function defined on $\Omega$, its directional derivative along $r_{i}$ at $u$ is denoted by

$$
r_{i} \bullet \phi(u) \doteq[\nabla \phi(u)] r_{i}(u)=\lim _{\epsilon \rightarrow 0} \frac{\phi\left(u+\epsilon r_{i}(u)\right)-\phi(u)}{\epsilon} .
$$

The differential of the $i$-th eigenvalue of the matrix $A\left(u^{-}, u^{+}\right)$w.r.t. the left and right states will be written as

$$
D \lambda_{i}\left(u^{+}, u^{-}\right) \cdot\left(U^{+}, U^{-}\right) \doteq \lim _{\epsilon \rightarrow 0} \frac{\lambda_{i}\left(u^{+}+\epsilon U^{+}, u^{-}+\epsilon U^{-}\right)-\lambda_{i}\left(u^{+}, u^{-}\right)}{\epsilon} .
$$

A similar notation is used for the differentials of the right and left eigenvectors of $A$.

For each $k \in\{1, \ldots, n\}$, we assume that either the $k$-th characteristic field is genuinely nonlinear and

$$
\lambda_{k}\left(u^{+}\right)+\varepsilon_{1}\left|u^{+}-u^{-}\right|<\lambda_{k}\left(u^{+}, u^{-}\right)<\lambda_{k}\left(u^{-}\right)-\varepsilon_{1}\left|u^{+}-u^{-}\right|
$$

for some $\varepsilon_{1}>0$ and all $u^{+}, u^{-} \in \Omega$ connected by an admissible shock of the $k$-th family, or that the $k$-th characteristic field is linearly degenerate, so that $r_{k} \bullet \lambda_{k}(u) \equiv 0$ and

$$
\lambda_{k}\left(u^{+}\right)=\lambda_{k}\left(u^{+}, u^{-}\right)=\lambda_{k}\left(u^{-}\right)
$$

whenever $u^{+}$and $u^{-}$are connected by a contact discontinuity of the $k$-th family.

For every fixed $k \in\{1, \ldots, m\}$, the couples of states $u^{+}, u^{-}$which are connected by a shock of the $k$-th characteristic family can be determined by the system of $n-1$ equations

$$
\left\langle l_{i}\left(u^{+}, u^{-}\right), u^{+}-u^{-}\right\rangle=0 \quad i \neq k .
$$

Of course, these correspond to the Rankine-Hugoniot equations

$$
F\left(u^{+}\right)-F\left(u^{-}\right)=A\left(u^{-}, u^{+}\right) \cdot\left(u^{+}-u^{-}\right)=\lambda_{k}\left(u^{-}, u^{+}\right) \cdot\left(u^{+}-u^{-}\right) .
$$


Differentiating (2.6) w.r.t. $u^{+}, u^{-}$, one obtains the system

$$
\Phi_{i}\left(u^{-}, u^{+}, U^{-}, U^{+}\right)=0 \quad i \neq k,
$$

where

$$
\begin{gathered}
\Phi_{i}\left(u^{-}, u^{+}, U^{-}, U^{+}\right) \doteq \sum_{j=1}^{n}\langle \\
\left.D l_{i}\left(u^{+}, u^{-}\right) \cdot\left(U_{j}^{+} r_{j}^{+}, U_{j}^{-} r_{j}^{-}\right), u^{+}-u^{-}\right\rangle \\
+\sum_{j=1}^{n}\left\langle l_{i}\left(u^{+}, u^{-}\right), U_{j}^{+} r_{j}^{+}-U_{j}^{-} r_{j}^{-}\right\rangle .
\end{gathered}
$$

Here $r_{j}^{-}=r_{j}\left(u^{-}\right), r_{j}^{+}=r_{j}\left(u^{+}\right)$. To express the general solution of (2.8), define the sets $\mathcal{I}$ and $\mathcal{O}$ (incoming and outgoing) of signed indices

$$
\begin{aligned}
& \mathcal{I} \doteq\left\{i^{+} ; i \leq k\right\} \cup\left\{i^{-} ; \quad i \geq k\right\} \\
& \mathcal{O} \doteq\left\{j^{-} ; j<k\right\} \cup\left\{j^{+} ; \quad j>k\right\}
\end{aligned}
$$

if the $k$-th characteristic field is genuinely nonlinear, while

$$
\mathcal{I} \doteq\left\{i^{+} ; i<k\right\} \cup\left\{i^{-} ; i>k\right\},
$$

in the linearly degenerate case. Observe that the system of $n-1$ scalar equations (2.8) is linear homogeneous w.r.t. the "first order variations" $U^{-}, U^{+}$, with coefficients which depend continuously on $u^{-}, u^{+}$. When $u^{-}=u^{+}$one has

$$
\frac{\partial \Phi_{i}}{\partial U_{j}^{ \pm}}= \pm \delta_{i j}
$$

Therefore, if $u^{-}$and $u^{+}$are sufficiently close to each other, one has

$$
\operatorname{det}\left(\frac{\partial \Phi_{i}\left(u^{-}, u^{+}, U^{-}, U^{+}\right)}{\partial U_{j}^{ \pm}}\right) \neq 0 \quad\left(i \neq k, j^{ \pm} \in \mathcal{O}\right) .
$$

In turn, when the $(n-1) \times(n-1)$ determinant in (2.12) does not vanish, one can solve (2.8) for the $n-1$ outgoing variables $U_{j}^{ \pm}, \quad j^{ \pm} \in \mathcal{O}$ :

$$
U_{j}^{ \pm}=\widetilde{U}_{j}\left(u^{-}, u^{+} ; U^{\mathcal{I}}\right) \quad j \in \mathcal{O}
$$

Here $U^{\mathcal{I}}$ denotes the set of $n+1$ incoming variables $\left\{U_{i}^{ \pm} ; i^{ \pm} \in \mathcal{I}\right\}$. We remark that, in the case where the $k$-th characteristic field is linearly degenerate, one has

$$
\frac{\partial \Phi_{i}}{\partial U_{k}^{ \pm}} \equiv 0
$$

hence all functions $\widetilde{U}_{j^{ \pm}}$in $(2.13)$ do not depend on $U_{k}^{+}, U_{k}^{-}$. This is consistent with our definition (2.11) of incoming waves.

Next, we discuss the boundary conditions. Let $(u, z) \in \mathbb{R}^{n} \times Z$ be a point such that the $n-p$ equations (1.3) hold. Keep $z$ fixed and consider an infinitesimal perturbation of $u$, having the form $u+\varepsilon \sum v_{i} r_{i}(u)$. We have

$$
\frac{d}{d \varepsilon} \phi_{j}\left(u+\varepsilon \sum_{i=1}^{n} v_{i} r_{i}(u), z\right)_{\varepsilon=0}=0 \quad j=1, \ldots, n-p
$$


if and only if the components $v_{i}$ of the perturbation satisfy the set of linear equations

$$
\sum_{i=1}^{n}\left(r_{i} \bullet \phi_{j}\right) v_{i}=0 \quad j=1, \ldots, n-p,
$$

where

$$
r_{i} \bullet \phi_{j}(u, z) \doteq \lim _{\epsilon \rightarrow 0} \frac{\phi_{j}\left(u+\epsilon r_{i}(u), z\right)-\phi_{j}(u, z)}{\epsilon} \quad j=1, \ldots, n-p .
$$

In the following, we assume that the linear homogeneous system (2.15) can be uniquely solved for the outgoing components $v^{\mathcal{O}}=\left(v_{p+1}, \ldots, v_{n}\right)$, as functions of the incoming components $v^{\mathcal{I}}=$ $\left(v_{1}, \ldots, v_{p}\right)$. This is the case if and only if the relevant $(n-p) \times(n-p)$ matrix of directional derivatives has non-zero determinant:

$$
\operatorname{det}\left(r_{i} \bullet \phi_{j}\right)_{\substack{i=p+1, \ldots, n \\ j=1, \ldots, n-p}} \neq 0 \text {. }
$$

The solution of $(2.15)$ can then be written in the form

$$
v_{j}=\widetilde{V}^{j}\left(u, z ; v_{1}, \ldots, v_{p}\right) \quad j=p+1, \ldots, n,
$$

where $\widetilde{V}_{j}$ is a linear function of the incoming components $v_{1}, \ldots, v_{p}$.

Under the same assumption (2.16), we can vary the control $z$, keep the incoming components unchanged, and uniquely determine the change in the outgoing components. More precisely, for any reference values $u, z$ and increment $z^{\prime}$, consider the linear system of equations

$$
\frac{d}{d \varepsilon} \phi_{j}\left(u+\varepsilon \sum_{i=p+1}^{n} v_{i} r_{i}(u), z+\varepsilon z^{\prime}\right)_{\varepsilon=0}=0 \quad j=1, \ldots, n-p .
$$

These are solved if and only if

$$
\frac{\partial \phi_{j}}{\partial z} \cdot z^{\prime}+\sum_{i=p+1}^{n}\left(r_{i} \bullet \phi_{j}\right) v_{i}=0 \quad j=1, \ldots, n-p .
$$

Assuming that the determinant in (2.16) does not vanish, the above linear system of $n-p$ equations can again be solved for the outgoing components $v_{p+1}, \ldots, v_{n}$, namely

$$
v_{j}=\Lambda_{j}(u, z) \cdot z^{\prime} \quad j=p+1, \ldots, n,
$$

for suitable linear mappings $\Lambda_{j}: \mathbb{R}^{m} \mapsto \mathbb{R}$.

In the following, we say that $u=u(t, x)$ is a piecewise $\mathcal{C}^{1}$ solution of the system of balance laws (1.1) if there exists finitely many $\mathcal{C}^{1}$ curves

$$
\gamma_{\alpha} \doteq\left\{(t, x) ; \quad x=x_{\alpha}(t), \quad t \in\left[t_{\alpha}^{\prime}, t_{\alpha}^{\prime \prime}\right]\right\}
$$

in the $t$ - $x$-plane, such that

(i) The function $u$ is a continuously differentiable solution of (1.1) on the complement of the curves $\gamma_{\alpha}$. 
(ii) The right limits $u(t, 0+)$ exist at all except finitely many times $t$, and satisfy the boundary conditions (1.3).

(iii) Along each curve $x=x_{\alpha}(t)$, there exist the limits

$$
\left.u\left(t, x_{\alpha} \pm\right)=\lim _{x \rightarrow x_{\alpha}(t) \pm} u(t, x), \quad u_{x}\left(t, x_{\alpha} \pm\right)=\lim _{x \rightarrow x_{\alpha}(t) \pm} u_{x}(t, x) \quad t \in\right] t_{\alpha}^{\prime}, t_{\alpha}^{\prime \prime}[.
$$

For some $k_{\alpha} \in\{1, \ldots, n\}$, the Rankine-Hugoniot conditions (1.7) hold, with shock speed

$$
\dot{x}_{\alpha}(t)=\lambda_{k_{\alpha}}\left(u\left(t, x_{\alpha}-\right), u\left(t, x_{\alpha}+\right)\right) .
$$

This speed satisfies the Lax admissibility conditions

$$
\lambda_{k_{\alpha}}\left(\left(u\left(t, x_{\alpha}-\right)\right) \geq \dot{x}_{\alpha}(t) \geq \lambda_{k_{\alpha}}\left(\left(u\left(t, x_{\alpha}+\right)\right) .\right.\right.
$$

The existence, uniqueness and continuous dependence of BV solutions to the initial-boundary value problem (1.1)-(1.3) has been the subject of an extensive literature and is now fairly well understood $[\mathrm{B}, \mathrm{D}, \mathrm{HR}]$. Conditions which guarantee that the solution remains smooth for all times are also known, although under very restrictive hypotheses [Li].

In the following, we say that $u$ has a weak discontinuity along $x_{\alpha}$ if $u_{x}$ is discontinuous but the function $u$ itself is continuous at each point $\left(t, x_{\alpha}(t)\right)$. In the case $u\left(t, x_{\alpha}+\right) \neq u\left(t, x_{\alpha}-\right)$, we say that $u$ has a strong discontinuity, or a jump, at $x_{\alpha}$.

\section{3 - Generalized tangent vectors.}

Let $u: \mathbb{R}_{+} \mapsto \mathbb{R}^{n}$ be a piecewise Lipschitz continuous function with discontinuities at points $0<x_{1}<\ldots<x_{N}$. Following [BM1], we define the space $T_{u}$ of generalized tangent vectors to $u$ as the Banach space $\mathbf{L}^{1}\left(\mathbb{R}_{+} ; R^{n}\right) \times \mathbb{R}^{N}$. On the family $\Sigma_{u}$ of all continuous paths $\gamma:\left[0, \varepsilon_{0}\right] \mapsto \mathbf{L}^{1}$ with $\gamma(0)=u$ (with $\varepsilon_{0}>0$ possibly depending on $\gamma$ ), consider the equivalence relation $\sim$ defined by

$$
\gamma \sim \gamma^{\prime} \Longleftrightarrow \lim _{\varepsilon \rightarrow 0} \frac{\left\|\gamma(\varepsilon)-\gamma^{\prime}(\varepsilon)\right\|_{\mathbf{L}^{1}}}{\varepsilon}=0 .
$$

We say that a continuous path $\gamma \in \Sigma_{u}$ generates the tangent vector $(v, \xi) \in T_{u}$ if $\gamma$ is equivalent to the path $\gamma_{(v, \xi ; u)}$ defined as

$$
\gamma_{(v, \xi ; u)}(\varepsilon)=u+\varepsilon v+\sum_{\xi_{\alpha}<0}\left(u\left(x_{\alpha}^{+}\right)-u\left(x_{\alpha}^{-}\right)\right) \chi_{\left[x_{\alpha}+\varepsilon \xi_{\alpha}, x_{\alpha}\right]}-\sum_{\xi_{\alpha}>0}\left(u\left(x_{\alpha}^{+}\right)-u\left(x_{\alpha}^{-}\right)\right) \chi_{\left[x_{\alpha}, x_{\alpha}+\varepsilon \xi_{\alpha}\right]} .
$$

Here $\chi_{[a, b]}$ denotes the characteristic function of the interval $[a, b]$. Up to higher order terms, $\gamma(\varepsilon)$ is thus obtained from $u$ by adding $\varepsilon v$ and shifting each point $x_{\alpha}$, where $u$ has a jump, by $\varepsilon \xi_{\alpha}$. In order to derive an evolution equation satisfied by these tangent vectors, one needs to consider more regular paths $\gamma \in \Sigma_{u}$, taking values within the set of piecewise Lipschitz functions. The following definitions are a natural extension of the ones given in [BM1], in connection with the more general initial-boundary value problem (1.1)-(1.3). 
Definition 1. Given the system of balance laws (1.1) and the boundary conditions (1.3), we say that a function $u: \mathbb{R}_{+} \mapsto \mathbb{R}^{n}$ is in the class PLSD of Piecewise Lipschitz functions with Simple Discontinuities if it satisfies the following conditions.

(i) $u$ has finitely many discontinuities, say at $0<x_{1}<x_{2}<\cdots<x_{N}$, and there exists a constant $L$ such that

$$
\left|u(x)-u\left(x^{\prime}\right)\right| \leq L\left|x-x^{\prime}\right|
$$

whenever the interval $\left[x, x^{\prime}\right]$ does not contain any point $x_{\alpha}$.

(ii) Each jump of $u$ consists of a contact discontinuity or of a single, stable shock. More precisely, for every $\alpha \in\{1, \ldots, N\}$, there exists $k_{\alpha} \in\{1, \ldots, m\}$ such that

$$
\begin{array}{ccc}
\left\langle l_{i}\left(u^{+}, u^{-}\right), u^{+}-u^{-}\right\rangle & =0 & \forall i \neq k_{\alpha}, \\
u^{+} \neq u^{-}, & \lambda_{k_{\alpha}}\left(u^{+}\right) & \leq \lambda_{k_{\alpha}}\left(u^{+}, u^{-}\right) \leq \lambda_{k_{\alpha}}\left(u^{-}\right),
\end{array}
$$

where $u^{+}, u^{-}$denote respectively the right and left limits of $u(x)$ as $x \rightarrow x_{\alpha}$.

(iii) The right limit $u(0+)$ satisfies the boundary conditions (1.3).

Definition 2. Let $u: \mathbb{R}_{+} \mapsto \mathbb{R}^{n}$ be a PLSD function. A path $\gamma \in \Sigma_{u}$ is a Regular Variation for $u$ if, for $\varepsilon \in\left[0, \varepsilon_{0}\right]$, all functions $u^{\varepsilon} \doteq \gamma(\varepsilon)$ are in PLSD, with jumps at points $x_{1}^{\varepsilon}<\ldots<x_{N}^{\varepsilon}$ depending continuously on $\varepsilon$. They all satisfy Definition 1 with a Lipschitz constant $L$ independent of $\varepsilon$.

For each $\varepsilon \in\left[0, \varepsilon_{0}\right]$, let $u^{\varepsilon}=u^{\varepsilon}(t, x)$ be a piecewise $\mathcal{C}^{1}$ solution of initial-boundary value problem (1.1)-(1.3), with jumps at $x_{1}^{\varepsilon}<\cdots<x_{N}^{\varepsilon}(t)$. Assume that, at some initial time $\bar{t}$, the family $u^{\varepsilon}(\bar{t}, \cdot)$ is a Regular Variation of $u(\bar{t}, \cdot)$, generating the tangent vector $(\bar{v}, \bar{\xi}) \in \mathbf{L}^{1} \times \mathbb{R}^{N}$. The following questions arise naturally:

- At a later time $t>\bar{t}$, is the family of solutions $u^{\varepsilon}(t, \cdot)$ still a Regular Variation of $u(t, \cdot)$ ?

- Can one write a linearized evolution equation, describing how the tangent vectors $(v, \xi)$ change in time?

For solutions defined on the whole real line, the detailed analysis carried out in [BM1] has shown that both of these questions have a positive answer, provided that the reference solution $u$ has a fixed number of non-interacting shocks. Moreover, a first order tangent vector can be defined also beyond a time where two shocks interact. In the following, we state the corresponding results for the initial-boundary value problem.

Proposition 1. Let $u=u(t, x)$ be a piecewise Lipschitz solution of the system (1.1)-(1.3), corresponding to Lipschitz continuous control functions $w, z$. Let $\left\{u^{\varepsilon} ; \varepsilon \geq 0\right\}$ be a family of solutions which provides a Regular Variation of $u$ at time $t=\bar{t}$ generating the tangent vector $(v, \xi) \in \mathbf{L}^{1} \times \mathbb{R}^{N}$. Assume that the discontinuities in $u^{\varepsilon}$ do not interact and do not touch the boundary. Moreover, assume that the Lipschitz constants of the $u^{\varepsilon}$ (outside the jumps) remain uniformly bounded. Then for $t>\bar{t}$ the family $u^{\varepsilon}(t, \cdot)$ is still a Regular Variation of $u(t, \cdot)$ and generates a generalized tangent vector $(v(t), \xi(t))$. This vector can be determined as the unique broad solution of the following linearized initial-boundary value problem:

$$
v_{t}+A(u) v_{x}+[D A(u) \cdot v] u_{x}=D_{u} g(x, u, w) \cdot v
$$


outside the discontinuities of $u$, supplemented by the initial data

$$
(v, \xi)(\bar{t})=(\bar{v}, \bar{\xi})
$$

by the boundary conditions at $x=0$

$$
D_{u} \phi_{j}(u(t), z(t)) \cdot v(t)=0 \quad j=1, \ldots, n-p,
$$

and by the jump conditions

$$
\begin{aligned}
\left\langle D l_{i}\left(u^{+}, u^{-}\right) \cdot\right. & \left.\left(\xi_{\alpha} u_{x}^{+}+v^{+}, \xi_{\alpha} u_{x}^{-}+v^{-}\right),\left(u^{+}-u^{-}\right)\right\rangle \\
+ & \left\langle l_{i}\left(u^{+}, u^{-}\right), \xi_{\alpha} u_{x}^{+}+v^{+}-\xi_{\alpha} u_{x}^{-}-v^{-}\right\rangle=0, \quad \forall i \neq k_{\alpha}, \\
\dot{\xi}_{\alpha}=D \lambda_{k_{\alpha}}\left(u^{+}, u^{-}\right) \cdot\left(\xi_{\alpha} u_{x}^{+}+v^{+}, \xi_{\alpha} u_{x}^{-}+v^{-}\right), &
\end{aligned}
$$

along each line $x=x_{\alpha}(t)$ where $u$ suffers a discontinuity in the $k_{\alpha}$-th characteristic family.

We recall that a broad solution to a semilinear hyperbolic system is a locally integrable function whose components satisfy the appropriate integral equations along almost all characteristics. For details we refer to $[\mathrm{B}, \mathrm{RY}]$.

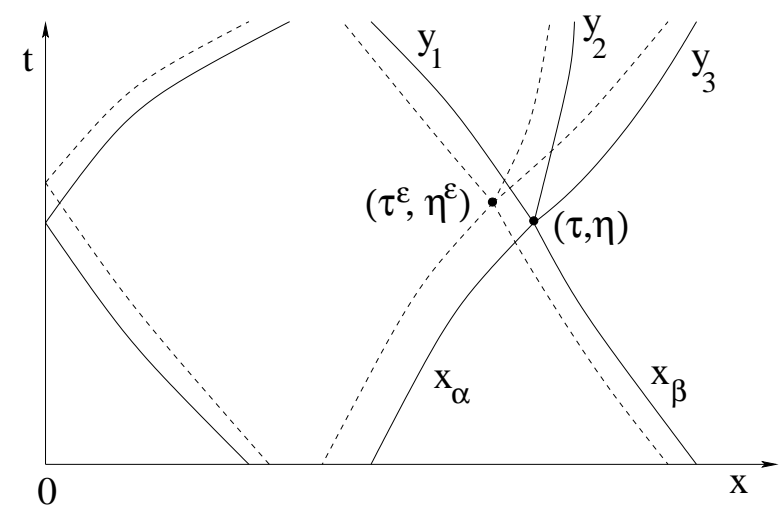

Figure 1

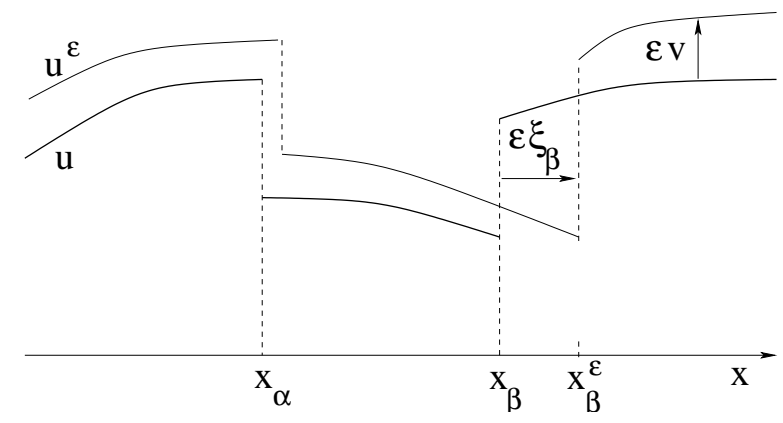

Figure 2 
The next result, also proved in [BM1], is concerned with the existence of generalized tangent vectors after a time $\tau$ where two shocks interact in the reference solution. To fix the ideas, let $x_{\alpha}(t)<x_{\beta}(t), t \leq \tau$, be the position of the shocks before interaction. Call $\eta=x_{\alpha}(\tau)=x_{\beta}(\tau)$ the interaction point. In a forward neighborhood of the point $(\tau, \eta)$, the reference solution $u$ is asymptotically close to the solution $\omega$ of the Riemann problem

$$
\omega_{t}+F(\omega)_{x}=0 \quad \omega(0, x)=\left\{\begin{array}{lll}
u(\tau, \eta-) & \text { if } \quad x<0 \\
u(\tau, \eta+) & \text { if } \quad x>0
\end{array}\right.
$$

in the sense that, for any $M$,

$$
\lim _{h \rightarrow 0+} \int_{-M h}^{M h}|u(\tau+h, \eta+x)-\omega(h, x)| d x=0 .
$$

To fix the ideas, assume that $\omega$ is piecewise continuous outside jumps occurring along the lines $x=\lambda_{i} t, i \in \mathcal{J} \subseteq\{1, \ldots, n\}$. Calling $N^{\prime}=|\mathcal{J}|$ the cardinality of the set of indices $\mathcal{J}$, after the interaction the solution will thus contain $N-2+N^{\prime}$ jumps.

Let us now consider the perturbed solutions $u^{\varepsilon}$. Call $\dot{x}_{\alpha}^{-}=\dot{x}_{\alpha}(\tau-), \dot{x}_{\beta}^{-}=\dot{x}_{\beta}(\tau-)$ the speeds of the incoming shocks at the interaction time, and let $\xi_{\alpha}^{-}=\xi_{\alpha}(\tau-), \xi_{\beta}^{-}=\xi_{\beta}(\tau-)$ be their shifts. In the perturbed solution $u^{\varepsilon}$, the shock interaction will take place at the point

$$
\left(\tau^{\varepsilon}, \eta^{\varepsilon}\right)=(\tau, \eta)+\varepsilon\left(\frac{\xi_{\beta}^{-}-\xi_{\alpha}^{-}}{\dot{x}_{\alpha}^{-}-\dot{x}_{\beta}^{-}}, \frac{\dot{x}_{\alpha}^{-} \xi_{\beta}^{-}-\dot{x}_{\beta}^{-} \xi_{\alpha}^{-}}{\dot{x}_{\alpha}^{-}-\dot{x}_{\beta}^{-}}\right)+o(\varepsilon) .
$$

The next proposition relates the tangent vector $(v(t), \xi(t))$ for $t>\tau$ after the interaction to the tangent vector $\left(v^{-}, \xi^{-}\right) \doteq(v(\tau-), \xi(\tau-))$ before the interaction. For $j \in \mathcal{J}$, we shall call $y_{j}$, $\hat{\xi}_{j}$ respectively the positions and the shift rates of the jumps emerging from the interaction point.

Proposition 2. In the same setting as Proposition 1, assume that in the reference solution two jumps $x_{\alpha}, x_{\beta}$ interact at time $\tau$, with $x_{\alpha}^{-}>x_{\beta}^{-}$. Then for $t>\tau$ the family of solutions $u^{\varepsilon}$ still generates a generalized tangent vector $(v(t), \xi(t)) \in \mathbf{L}^{1} \times \mathbb{R}^{N-2+N^{\prime}}$. This is related to $\left(v^{-}, \xi^{-}\right)$by the following formulas. Let $M$ be an upper bound for all characteristic speeds, so that $\left|\lambda_{i}(u)\right| \leq M$ for all $u$. Then

$$
\begin{gathered}
\lim _{t \rightarrow \tau+} \int_{|x-\eta|>M(t-\tau)}\left|v(t, x)-v^{-}(x)\right| d x=0, \\
\lim _{t \rightarrow \tau+} \int_{|x-\eta|<M(t-\tau)}\left|v(t, x)+\frac{\xi_{\beta}^{-}-\xi_{\alpha}^{-}}{\dot{x}_{\alpha}^{-}-\dot{x}_{\beta}^{-}} \cdot \omega_{t}(t, x)+\frac{\dot{x}_{\alpha}^{-} \xi_{\beta}^{-}-\dot{x}_{\beta}^{-} \xi_{\alpha}^{-}}{\dot{x}_{\alpha}^{-}-\dot{x}_{\beta}^{-}} \cdot \omega_{x}(t, x)\right| d x=0, \\
\lim _{t \rightarrow \tau+} \xi_{\gamma}(t)=\xi_{\gamma}^{-} \quad \gamma \neq \alpha, \beta, \\
\lim _{t \rightarrow \tau+} \hat{\xi}_{j}(t)=\frac{\dot{x}_{\alpha}^{-} \xi_{\beta}^{-}-\dot{x}_{\beta}^{-} \xi_{\alpha}^{-}}{\dot{x}_{\alpha}^{-}-\dot{x}_{\beta}^{-}}-\frac{\xi_{\beta}^{-}-\xi_{\alpha}^{-}}{\dot{x}_{\alpha}^{-}-\dot{x}_{\beta}^{-}} \cdot \dot{y}_{j}^{+} \quad j \in \mathcal{J} .
\end{gathered}
$$

Remark 1. The first two limits describe the behavior of the continuous part of the tangent vector $(v, \xi)$ immediately after the interaction has taken place. Away from the point of interaction, according to (3.13) the function $v$ does not change across the interaction time $\tau$. In a small neighborhood of the interaction point, for $t>\tau$ the $v$-component of the tangent vector has to 
account for the shift in space and time of the solution $\omega$ of the Riemann problem. This is reflected in the last two terms inside the integral in (3.14). The last two limits in Proposition 2 describe the behavior of the jump part. By (3.15), the shifts in the jumps at points $x_{\gamma}$ away from the interaction do not change across the interaction time. The shifts of the new shocks emerging from the interaction, according to (3.16), depends on their speeds $\dot{y}_{j}$ and on the shift in space and in time of the interaction point.

By similar arguments, one can prove the existence of generalized tangent vectors after a time $\tau$ where one of the shock hits the boundary, in the reference solution. To fix the ideas, let $x_{\alpha}(t)>0$ be the position of the shock for $t<\tau$, so that $x_{\alpha} \rightarrow 0$ as $t \rightarrow \tau-$. We assume that the boundary control $z(\cdot)$ is continuous at $t=\tau$. In a forward neighborhood of the point $(\tau, 0)$, the reference solution $u$ is asymptotically close to the solution $\tilde{\omega}: \mathbb{R}_{+} \times \mathbb{R}_{+} \mapsto \mathbb{R}^{n}$ of the boundary Riemann problem

$$
\begin{gathered}
\tilde{\omega}_{t}+F(\tilde{\omega})_{x}=0 \\
\begin{cases}\tilde{\omega}(0, x)=u(\tau, 0+) & \text { for } x>0, \\
\phi_{j}(\tilde{\omega}(t, 0), z(\tau))=0 & j=1, \ldots, n-p, \text { for } t>0 .\end{cases}
\end{gathered}
$$

More precisely, for $M$ arbitrarily large there holds

$$
\lim _{h \rightarrow 0+} \int_{0}^{M h}|u(\tau+h, y+x)-\tilde{\omega}(h, x)| d x=0 .
$$

To fix the ideas, assume that $\tilde{\omega}$ is piecewise continuous outside jumps occurring along the lines $x=\lambda_{i} t, i \in \mathcal{J} \subseteq\{p+1, \ldots, n\}$. Notice that $i>p$ because these lines must have positive speed. Calling $N^{\prime}=|\mathcal{J}|$ the cardinality of the set of indices $\mathcal{J}$, after the interaction the solution will thus contain $N-1+N^{\prime}$ jumps.

Let us now consider the perturbed solutions $u^{\varepsilon}$. Call $\dot{x}_{\alpha}^{-}=\dot{x}_{\alpha}(\tau-)<0$ the speed of the incoming shock at the interaction time, and let $\xi_{\alpha}^{-}=\xi_{\alpha}(\tau-)$ be its shift. In the perturbed solution $u^{\varepsilon}$, the shock will hit the boundary at the time

$$
\tau^{\varepsilon}=\tau-\varepsilon \frac{\xi_{\alpha}^{-}}{\dot{x}_{\alpha}^{-}}+o(\varepsilon)
$$

The next proposition relates the tangent vector $(v(t), \xi(t))$ for $t>\tau$ after the shock hits the boundary to the previous tangent vector $\left(v^{-}, \xi^{-}\right) \doteq(v(\tau-), \xi(\tau-))$. For $j \in \mathcal{J}$, we shall call $y_{j}$, $\hat{\xi}_{j}$ respectively the positions and the shift rates of the jumps emerging from the interaction point.

Proposition 3. In the same setting as Proposition 1, assume that in the reference solution the jump $x_{\alpha}$ hits the boundary at time $\tau$. Then for $t>\tau$ the family of solutions $u^{\varepsilon}$ still generates a generalized tangent vector $(v(t), \xi(t)) \in \mathbf{L}^{1} \times \mathbb{R}^{N-1+N^{\prime}}$. This is related to $\left(v^{-}, \xi^{-}\right)$by the following formulas. Let $M$ be an upper bound for all characteristic speeds, so that $\left|\lambda_{i}(u)\right| \leq M$ for all $u$. Then

$$
\begin{gathered}
\lim _{t \rightarrow \tau+} \int_{M(t-\tau)}^{\infty}\left|v(t, x)-v^{-}(x)\right| d x=0, \\
\lim _{t \rightarrow \tau+} \int_{0}^{M(t-\tau)}\left|v(t, x)-\frac{\xi_{\alpha}^{-}}{\dot{x}_{\alpha}^{-}} \cdot \tilde{\omega}_{t}(t, x)\right| d x=0, \\
\lim _{t \rightarrow \tau+} \xi_{\gamma}(t)=\xi_{\gamma}^{-} \quad \gamma \neq \alpha,
\end{gathered}
$$




$$
\lim _{t \rightarrow 0+} \hat{\xi}_{j}(t)=-\frac{\xi_{\alpha}^{-}}{\dot{x}_{\alpha}^{-}} \cdot \dot{y}_{j}^{+} \quad j \in \mathcal{J}
$$

It is often useful to rewrite the equations (3.6) and (3.8)-(3.10) in terms of the scalar components $u_{x}^{i}=\left\langle l_{i}(u), u_{x}\right\rangle, v_{i}=\left\langle l_{i}(u), v\right\rangle$. We recall that $l_{i}(u)$ denotes the $i$-th left eigenvector of the Jacobian matrix $A(u)=D F(u)$. Differentiating w.r.t. $\varepsilon$ the equation

$$
A(u+\varepsilon v) u_{x}=\sum_{i=1}^{n} \lambda_{i}(u+\varepsilon v)\left\langle l_{i}(u+\varepsilon v), u_{x}\right\rangle r_{i}(u+\varepsilon v),
$$

at $\varepsilon=0$ one obtains

$$
[D A(u) \cdot v] u_{x}=\sum_{i, j}\left(r_{j} \bullet \lambda_{i}\right) u_{x}^{i} v_{j} r_{i}+\sum_{i, j} \lambda_{i}\left\langle r_{j} \bullet l_{i}, u_{x}\right\rangle v_{j} r_{i}+\sum_{i, j} \lambda_{i} u_{x}^{i}\left(r_{j} \bullet r_{i}\right) v_{j}
$$

Combining (3.22) with the equations

$$
\begin{array}{cc}
l_{i, t}=D_{u} l_{i} \cdot u_{t}=\sum_{j}\left(r_{j} \bullet l_{i}\right)\left\langle l_{j}, u_{t}\right\rangle=\sum_{j}\left(r_{j} \bullet l_{i}\right)\left(-\lambda_{j} u_{x}^{j}+\left\langle l_{j}, g\right\rangle\right), \\
l_{i, x}=\sum_{j}\left(r_{j} \bullet l_{i}\right) u_{x}^{j}, \quad \lambda_{i, x}=\sum_{j}\left(r_{j} \bullet \lambda_{i}\right) u_{x}^{j}, \\
\left\langle r_{j} \bullet l_{i}, r_{k}\right\rangle+\left\langle l_{i}, r_{j} \bullet r_{k}\right\rangle=r_{j} \bullet\left\langle l_{i}, r_{k}\right\rangle \equiv 0,
\end{array}
$$

and multiplying (3.6) on the left by $l_{i}$ we find

$$
\begin{aligned}
\left(v_{i}\right)_{t}+\left(\lambda_{i} v_{i}\right)_{x}+\sum_{k \neq i}\left(r_{k} \bullet \lambda_{i}\right)\left\{u_{x}^{i} v_{k}-u_{x}^{k} v_{i}\right\}+\sum_{j \neq k}\left\langle l_{i},\left[r_{j}, r_{k}\right]\right\rangle\left(\lambda_{i}-\lambda_{j}\right) u_{x}^{j} v_{k} \\
=-\sum_{j, k}\left\langle l_{i}, r_{j} \bullet r_{k}\right\rangle \cdot\left\langle l_{j}, g\right\rangle v_{k}+\sum_{k}\left\langle l_{i}, r_{k} \bullet g\right\rangle v_{k} \quad(i=1, \ldots, n) .
\end{aligned}
$$

Here $\left[r_{j}, r_{k}\right] \doteq r_{j} \bullet r_{k}-r_{k} \bullet r_{j}$ denotes the Lie bracket of the vector fields $r_{j}, r_{k}$.

Concerning the equations (3.8), fix a shock curve $t \mapsto x_{\alpha}(t)$ and call $u^{-}, u^{+}$the limits of $u(t, x)$ as $x \rightarrow x_{\alpha}(t)$ from the left and from the right, respectively. Define the scalar components $v_{i}^{ \pm} \doteq\left\langle l_{i}\left(u^{ \pm}\right), v^{ \pm}\right\rangle$, so that $v^{+}=\sum r_{i}^{+} v_{i}^{+}, \quad v^{-}=\sum r_{i}^{-} v_{i}^{-}$. We now recognize that the system (3.9) is equivalent to (2.8), with $k=k_{\alpha}$ and $U^{ \pm}=\xi_{\alpha} u_{x}^{ \pm}+v^{ \pm}$. Given $\xi_{\alpha}, u_{x}^{ \pm}$and the values $v_{i}^{ \pm}$ $\left(i^{ \pm} \in \mathcal{I}\right)$ of the incoming components, the $n-1$ scalar equations (3.9) can be uniquely solved for the $n-1$ outgoing components:

$$
v_{j}^{ \pm}=V_{\alpha}^{j \pm}\left(v^{\mathcal{I}}, \xi_{\alpha}\right) \quad j^{ \pm} \in \mathcal{O} .
$$

Observe that the $V_{\alpha}^{j \pm}$ are linear homogeneous functions of $\xi_{\alpha}$ and of the incoming variables $v^{\mathcal{I}}$. In turn, inserting these values in (3.10), one obtains an expression for the time derivative of the shift

$$
\dot{\xi}_{\alpha}=\Psi_{\alpha}\left(v^{\mathcal{I}}, \xi_{\alpha}\right)
$$

In an entirely similar way, the boundary equations (3.8) can be rewritten as a linear homogeneous system of $n-p$ scalar equations for the components $v_{i}(t, 0)=\left\langle l_{i}(u(t, 0)), v(t, 0)\right\rangle$. We can 
identify a set of $p$ incoming components (approaching the boundary) $\mathcal{I} \doteq\{1, \ldots, p\}$ and a set of $n-p$ outgoing components $\mathcal{O} \doteq\{p+1, \ldots, n\}$. The system can then be solved for the outgoing components, according to (2.17).

\section{4 - The adjoint equations}

Let the function $u: \mathbb{R} \mapsto \mathbb{R}^{n}$ be piecewise Lipschitz continuous with $N$ points of jump. We then define the space of generalized cotangent vectors (or adjoint vectors) to $u$ as the Banach space $T_{u}^{*} \doteq \mathbf{L}^{1}(\mathbb{R}) \times \mathbb{R}^{N}$. Elements of $T_{u}^{*}$ will be written as $\left(v^{*}, \xi^{*}\right)$ and regarded as row vectors.

Given a piecewise Lipschitz solution $u=u(t, x)$ of (1.1) and (1.3), with jumps along the lines $x=x_{\alpha}(t), \alpha=1, \ldots, N$, we seek an adjoint system of linear equations on $T_{u}^{*}$ whose solutions $\left(v^{*}(t, \cdot), \xi^{*}(t)\right)$ have the property that the duality product

$$
\left\langle\left(v^{*}, \xi^{*}\right),(v, \xi)\right\rangle \doteq \int_{0}^{\infty} v^{*}(t, x) \cdot v(t, x) d x+\sum_{\alpha=1}^{N} \xi_{\alpha}^{*}(t) \xi_{\alpha}(t)
$$

remains constant in time, for every solution $(v, \xi)$ of the linear system (3.6)-(3.10). At this stage, we assume that the jump curves $x_{\alpha}$ do not touch the boundary at $x=0$ and do not interact with each other.

Assume that (4.1) holds for every solution $v$ of (3.6) which vanishes on a neighborhood of all lines $x=x_{\alpha}(t)$. Then an integration by parts shows that, away from the discontinuities of $u$, the function $v^{*}$ must satisfy

$$
v_{t}^{*}+v_{x}^{*} A(u)=-v^{*} \cdot D_{u} g(x, u, w) .
$$

In order to formulate also a suitable set of boundary conditions, valid along the lines $x=x_{\alpha}(t)$, it is convenient to work with the components $u_{x}^{i}=\left\langle l_{i}(u), u_{x}\right\rangle, v_{i}^{*}=\left\langle v^{*}, r_{i}(u)\right\rangle$. For a fixed $\alpha$, we shall write $\lambda_{i}^{+} \doteq \lambda_{i}\left(u\left(x_{\alpha}+\right)\right)$ and $\lambda_{i}^{-} \doteq \lambda_{i}\left(u\left(x_{\alpha}-\right)\right)$ for the the $i$-th characteristic speeds to the right and to the left of the jump at $x_{\alpha}$, respectively. Similarly, we write $v_{i+}^{*} \doteq v_{i}^{*}\left(x_{\alpha}+\right), v_{i-}^{*} \doteq v_{i}^{*}\left(x_{\alpha}-\right)$. In the following, $V_{\alpha}^{j}, \Psi_{\alpha}$ are the linear functions introduced at (3.24)-(3.25). Moreover, $\widetilde{V}_{j}$ are the linear functions in (2.17).

Proposition 4. Let $u$ be a piecewise $\mathcal{C}^{1}$ solution of the hyperbolic system (1.1), with jumps occurring along the (nonintersecting) lines $x=x_{\alpha}(t)$. Assume that the map $t \mapsto\left(v^{*}(t, \cdot), \xi^{*}(t)\right) \in$ $T_{u}^{*}$, with $v^{*}=\sum l_{i}(u) v_{i}^{*}$, provides a solution to the linear system (4.2) outside the lines where $u$ is discontinuous, together with the equations

$$
\begin{aligned}
& \dot{\xi}_{\alpha}^{*}=-\frac{\partial \Psi_{\alpha}}{\partial \xi_{\alpha}} \cdot \xi_{\alpha}^{*}-\sum_{j^{ \pm} \in \mathcal{O}}\left|\lambda_{j}^{ \pm}-\dot{x}_{\alpha}\right| \frac{\partial V_{\alpha}^{j}}{\partial \xi_{\alpha}} \cdot v_{j \pm}^{*}, \\
& v_{i \pm}^{*}=\frac{1}{\left|\lambda_{i}^{ \pm}-\dot{x}_{\alpha}\right|}\left\{\frac{\partial \Psi_{\alpha}}{\partial v_{i \pm}} \cdot \xi_{\alpha}^{*}+\sum_{j^{ \pm} \in \mathcal{O}}\left|\lambda_{j}^{ \pm}-\dot{x}_{\alpha}\right| \frac{\partial V_{\alpha}^{j}}{\partial v_{i \pm}} \cdot v_{j \pm}^{*}\right\} \quad i^{ \pm} \in \mathcal{I},
\end{aligned}
$$

along each line $x=x_{\alpha}(t)$, and moreover it satisfies

$$
v_{i}^{*}=\sum_{j=p+1}^{n}\left|\frac{\lambda_{j}}{\lambda_{i}}\right| \frac{\partial \widetilde{V}^{j}}{\partial v_{i}} \cdot v_{j}^{*} \quad i=1, \ldots, p,
$$


along the boundary where $x=0$. Then, for every solution $(v, \xi)$ of (3.6)-(3.8), the product (4.1) remains constant in time.

Proof. For notational convenience, we set $x_{0}(t)=0, x_{N+1}(t)=+\infty$. We recall that, along the boundary $x=0$, the sets of incoming and outgoing indices are $\mathcal{I} \doteq\{1, \ldots, p\}$ and $\mathcal{O} \doteq$ $\{p+1, \ldots, n\}$. We observe that

$$
\begin{gathered}
\sum_{i} v_{i}^{*} v_{i}=\left(\sum_{i} v_{i}^{*} l_{i}(u)\right)\left(\sum_{j} v_{j} r_{j}(u)\right) \\
\sum_{i} \lambda_{i}(u) v_{i}^{*} v_{i}=\left(\sum_{i} \lambda_{i}(u) v_{i}^{*} l_{i}(u)\right)\left(\sum_{j} v_{j} r_{j}(u)\right)=v^{*} A(u) v .
\end{gathered}
$$

By (4.6), integrating each component $v_{i}^{*} v_{i}$ along the corresponding characteristic lines $\dot{x}=\lambda_{i}(u)$, the time derivative of (4.1) can now be computed as

$$
\begin{aligned}
& \frac{d}{d t}\left[\int \sum_{i} v_{i}^{*} v_{i} d x+\sum_{\alpha} \xi_{\alpha}^{*} \xi_{\alpha}\right]=\sum_{\alpha=0}^{N} \sum_{i} \int_{x_{\alpha}(t)}^{x_{\alpha+1}(t)}\left[\left(v_{i}^{*} v_{i}\right)_{t}+\left(\lambda_{i}(u) v_{i}^{*} v_{i}\right)_{x}\right] d x \\
& \quad+\sum_{\alpha=0}^{N}\left[\sum_{j^{ \pm} \in \mathcal{O}}\left|\lambda_{j}^{ \pm}-\dot{x}_{\alpha}\right| \cdot v_{j \pm}^{*} v_{j \pm}-\sum_{i^{ \pm} \in \mathcal{I}}\left|\lambda_{i}^{ \pm}-\dot{x}_{\alpha}\right| \cdot v_{i \pm}^{*} v_{i \pm}\right] \\
& \quad+\sum_{\alpha=1}^{N}\left(\dot{\xi}_{\alpha}^{*} \xi_{\alpha}+\xi_{\alpha}^{*} \dot{\xi}_{\alpha}\right)
\end{aligned}
$$

Recalling (4.7), from the linear equations (3.6) and (4.2) we deduce

$$
\sum_{i}\left(v_{i}^{*} v_{i}\right)_{t}+\sum_{i}\left(\lambda_{i}(u) v_{i}^{*} v_{i}\right)_{x}=0
$$

Therefore, the integrals on the right hand side of (4.8) vanishes.

Next, fix any jump curve, say $x_{\alpha}$ with $\alpha \in\{1, \ldots, N\}$. Recall that the functions $V_{\alpha}^{j}, \Psi_{\alpha}$ in (3.24)-(3.25) are linear homogeneous w.r.t. the variables $\xi_{\alpha}, v_{i^{ \pm}}, i^{ \pm} \in \mathcal{I}$. Therefore, we can write

$$
\dot{\xi}_{\alpha}=\frac{\partial \Psi_{\alpha}}{\partial \xi_{\alpha}} \cdot \xi_{\alpha}+\sum_{i^{ \pm} \in \mathcal{I}} \frac{\partial \Psi_{\alpha}}{\partial v_{i^{ \pm}}} \cdot v_{i^{ \pm}} \quad v_{j^{ \pm}}=\frac{\partial V_{\alpha}^{j}}{\partial \xi_{\alpha}} \cdot \xi_{\alpha}+\sum_{i^{ \pm} \in \mathcal{I}} \frac{\partial V_{\alpha}^{j}}{\partial v_{i^{ \pm}}} \cdot v_{i^{ \pm}} \quad j^{ \pm} \in \mathcal{O} .
$$

Using (4.10) and factoring out the terms $\xi_{\alpha}, v_{i^{ \pm}}$, the last two terms in (4.8) can be written as

$$
\begin{gathered}
{\left[\sum_{j^{ \pm} \in \mathcal{O}}\left|\lambda_{j}^{ \pm}-\dot{x}_{\alpha}\right| \cdot v_{j \pm}^{*} v_{j \pm}-\sum_{i^{ \pm} \in \mathcal{I}}\left|\lambda_{i}^{ \pm}-\dot{x}_{\alpha}\right| \cdot v_{i \pm}^{*} v_{i \pm}\right]+\sum_{\alpha=1}^{N}\left(\dot{\xi}_{\alpha}^{*} \xi_{\alpha}+\xi_{\alpha}^{*} \dot{\xi}_{\alpha}\right)} \\
=\sum_{i_{ \pm} \in \mathcal{I}}\left[\sum_{j^{ \pm} \in \mathcal{O}}\left|\lambda_{j}^{ \pm}-\dot{x}_{\alpha}\right| \frac{\partial V_{\alpha}^{j}}{\partial v_{i \pm}} v_{j \pm}^{*}-\left|\lambda_{i}^{ \pm}-\dot{x}_{\alpha}\right| \cdot v_{i \pm}^{*}+\frac{\partial \Psi_{\alpha}}{\partial v_{i^{ \pm}}} \xi_{\alpha}^{*}\right] \cdot v_{i \pm} \\
+\left[\dot{\xi}_{\alpha}^{*}+\xi_{\alpha}^{*} \cdot \frac{\partial \Psi_{\alpha}}{\partial \xi_{\alpha}}+\sum_{j^{ \pm} \in \mathcal{O}}\left|\lambda_{j}\left(u^{ \pm}\right)-\dot{x}_{\alpha}\right| v_{j^{ \pm}}^{*} \cdot \frac{\partial V_{\alpha}^{j}}{\partial \xi_{\alpha}}\right] \cdot \xi_{\alpha} .
\end{gathered}
$$


If the incoming components $v_{i \pm}^{*}, i^{ \pm} \in \mathcal{I}$ and $\dot{\xi}_{\alpha}^{*}$ satisfy the equations (4.3)-(4.4), the terms in square brackets on the right hand side of (4.11) vanish.

To complete the proof we need to consider separately what happens at the boundary $x_{0}=0$. In place of (4.11) we now have the simpler formula

$$
\begin{aligned}
& {\left[\sum_{j=p+1}^{n} \lambda_{j} \cdot v_{j \pm}^{*} v_{j \pm}+\sum_{i=1}^{p} \lambda_{i} \cdot v_{i \pm}^{*} v_{i \pm}\right]} \\
& =\sum_{i=1}^{p}\left[\sum_{j=p+1}^{n} \lambda_{j} \frac{\partial \widetilde{V}^{j}}{\partial v_{i}} v_{j}^{*}+\lambda_{i} \cdot v_{i}^{*}\right] \cdot v_{i \pm} .
\end{aligned}
$$

If the incoming components $v_{i}^{*}, i=1, \ldots, p$, satisfy the conditions (4.5), then the right hand side of (4.12) vanishes. This completes the proof of Proposition 4.

Remark 2. The equations (4.4)-(4.5) determine the incoming variables $v_{i^{ \pm}}^{*}, i^{ \pm} \in \mathcal{I}$, in terms of the outgoing variables $v_{j^{ \pm}}^{*}, j^{ \pm} \in \mathcal{O}$. Therefore, the Cauchy problem for the adjoint linear system (4.3)-(4.5) is well posed if one assigns the terminal values $\left(v^{*}(T, \cdot), \xi^{*}(T)\right)$ and seeks a solution defined backward in time.

Remark 3. If, at $x_{\alpha}$, the jump of $u$ consists of a contact discontinuity in the $k_{\alpha}$-th characteristic family, then the equations (4.4) determine only the $n-1$ incoming components $v_{i \pm}^{*}, i^{ \pm} \in \mathcal{I}$, with $\mathcal{I}$ defined by (2.14). In this case, the equations (4.10) still hold, because the functions $\Psi_{\alpha}, V_{\alpha}^{j}$ do not depend on $v_{k_{\alpha}^{ \pm}}$.

In the remainder of this section, we study what happens when two shocks interact in the reference solution. Our goal is to derive a formula, showing how generalized covectors can be prolonged backward in time, across the interaction. To fix the ideas, let $x=x_{\alpha}(t)$ and $x=x_{\beta}(t)$ be the interacting shock lines. All notations used in Proposition 2 will be retained here; in particular, $\tau, \eta$ are the time and location of the interaction, while $\omega=\omega(t, x)$ is the solution of the Riemann problem (3.11). As in (3.16), we write $\hat{\xi}_{j}(t), \hat{\xi}_{j}(t)^{*}, \quad(j \in \mathcal{J}, t>\tau)$ for the components of the tangent and cotangent vectors corresponding to the jumps in $u$ which originate from the point of interaction.

Let now $\left(v^{*}, \xi^{*}\right)$ be a solution of (4.2)-(4.5) defined for $t>\tau$. Fix a constant $M>0$ strictly larger than all wave speeds. Assume that the weak limit

$$
v^{*+} \doteq \mathrm{w}-\lim _{t \rightarrow \tau+} v^{*}(t, \cdot) \cdot \chi_{\{|x-\eta|>M(t-\tau)\}}
$$

exists, together with the limits

$$
\begin{gathered}
\hat{\xi}_{j}^{*+} \doteq \lim _{t \rightarrow \tau+} \hat{\xi}_{j}^{*}(t) \\
\Phi_{t}^{*} \doteq \lim _{t \rightarrow 0+} \int_{-M t}^{M t} v^{*}(\tau+t, \eta+x) \omega_{t}(t, x) d x, \quad \Phi_{x}^{*} \doteq \lim _{t \rightarrow 0+} \int_{-M t}^{M t} v^{*}(\tau+t, \eta+x) \omega_{x}(t, x) d x .
\end{gathered}
$$

Then, for every tangent vector $(v, \xi)$ satisfying (3.13)-(3.16), the duality product (4.1) will remain constant across the time $t=\tau$ provided that

$$
\mathrm{w}-\lim _{t \rightarrow \tau-} v^{*}(t)=v^{*+},
$$




$$
\lim _{t \rightarrow \tau-}\left(\xi_{\alpha}^{*}(t) \xi_{\alpha}^{-}+\xi_{\beta}^{*}(t) \xi_{\beta}^{-}\right)=\frac{\xi_{\alpha}^{-}-\xi_{\beta}^{-}}{\dot{x}_{\alpha}^{-}-\dot{x}_{\beta}^{-}} \cdot \Phi_{t}^{*}+\frac{\dot{x}_{\beta}^{-} \xi_{\alpha}^{-}-\dot{x}_{\alpha}^{-} \xi_{\beta}^{-}}{\dot{x}_{\alpha}^{-}-\dot{x}_{\beta}^{-}} \cdot \Phi_{x}^{*}+\sum_{j \in \mathcal{J}} \hat{\xi}_{j}^{*+} \hat{\xi}_{j}^{+} .
$$

Observing that the limits $\xi_{\alpha}^{-}, \xi_{\beta}^{-}$can be arbitrary, from (3.13)-(3.16) we obtain a set of equations relating the limits of the cotangent vector $\left(v^{*}, \xi^{*}\right)$ across the interaction time.

Proposition 5. In the same setting as Proposition 2, assume that the cotangent vector $\left(v^{*}, \xi^{*}\right)$ is prolonged backward across the interaction time according to (4.16), together with

$$
\begin{gathered}
\lim _{t \rightarrow \tau-} \xi_{\alpha}^{*}(t)=\frac{-1}{\dot{x}_{\alpha}^{-}-\dot{x}_{\beta}^{-}} \cdot \Phi_{t}^{*}+\frac{\dot{x}_{\beta}^{-}}{\dot{x}_{\alpha}^{-}-\dot{x}_{\beta}^{-}} \cdot \Phi_{x}^{*}-\sum_{j \in \mathcal{J}}\left[\frac{\dot{y}_{j}^{+}}{\dot{x}_{\alpha}^{-}-\dot{x}_{\beta}^{-}}+\frac{\dot{x}_{\beta}^{-}}{\dot{x}_{\alpha}^{-}-\dot{x}_{\beta}^{-}}\right] \hat{\xi}_{j}^{*+}, \\
\lim _{t \rightarrow \tau-} \xi_{\beta}^{*}(t)=\frac{1}{\dot{x}_{\alpha}^{-}-\dot{x}_{\beta}^{-}} \cdot \Phi_{t}^{*}-\frac{\dot{x}_{\alpha}^{-}}{\dot{x}_{\alpha}^{-}-\dot{x}_{\beta}^{-}} \cdot \Phi_{x}^{*}+\sum_{j \in \mathcal{J}}\left[\frac{\dot{y}_{j}^{+}}{\dot{x}_{\alpha}^{-}-\dot{x}_{\beta}^{-}}+\frac{\dot{x}_{\alpha}^{-}}{\dot{x}_{\alpha}^{-}-\dot{x}_{\beta}^{-}}\right] \hat{\xi}_{j}^{*+} . \\
\lim _{t \rightarrow \tau-} \xi_{\gamma}^{*}(t)=\lim _{t \rightarrow \tau+} \xi_{\gamma}^{*}(t) \quad \gamma \neq \alpha, \beta .
\end{gathered}
$$

Then, for every solution $(v, \xi)$ of (3.6)-(3.8), the product (4.1) remains constant in time, also across the interaction.

Indeed, by (4.15), the equations (4.18)-(4.19) imply the equality (4.17). Notice that all other components $\xi_{\gamma}^{*}$, corresponding to discontinuities in $u$ not involved in the interaction, will remain constant across the time $t=\tau$.

Together, the equations (4.16) and (4.18)-(4.20) determine the backward limit of the tangent vector $\left(v^{*}, \xi^{*}\right)$ in terms of its forward limit at time $\tau$. This allows us to prolong the solution of the adjoint system (4.2)-(4.5) backward in time.

We conclude this section by studying the case of a jump $x_{\alpha}$ hitting the boundary $x=0$ at time $t=\tau$. As in our previous analysis, call $\tilde{\omega}$ the solution of the boundary Riemann problem (3.17). To fix the ideas, assume that $\tilde{\omega}$ is piecewise continuous outside jumps occurring along the lines $x=\lambda_{i} t, i \in \mathcal{J} \subseteq\{p+1, \ldots, n\}$. We now assume that the weak limit

$$
v^{*+} \doteq \mathrm{w}_{t \rightarrow \tau+} \lim ^{*}(t, \cdot) \cdot \chi_{\{x>M(t-\tau)\}}
$$

exists, together with the limits

$$
\begin{gathered}
\hat{\xi}_{j}^{*+} \doteq \lim _{t \rightarrow \tau+} \hat{\xi}_{j}^{*}(t) \quad j \in \mathcal{J}, \\
\Phi_{t}^{*} \doteq \lim _{t \rightarrow 0+} \int_{0}^{M t} v^{*}(\tau+t, x) \tilde{\omega}_{t}(t, x) d x .
\end{gathered}
$$

In this case, the duality product (4.1) will remain constant across the interaction provided that

$$
\begin{gathered}
\mathrm{w}-\lim _{t \rightarrow \tau-} v^{*}(t)=v^{*+} \\
\lim _{t \rightarrow \tau-} \xi_{\alpha}^{*}(t) \xi_{\alpha}^{-}=\frac{\xi_{\alpha}^{-}}{\dot{x}_{\alpha}^{-}} \cdot \Phi_{t}^{*}+\sum_{j \in \mathcal{J}} \hat{\xi}_{j}^{*+} \hat{\xi}_{j}^{+} .
\end{gathered}
$$


Observing that the incoming component $\xi_{\alpha}$ can be arbitrary, we obtain a set of equations relating the limits of the cotangent vector $\left(v^{*}, \xi^{*}\right)$ across the interaction time.

Proposition 6. In the same setting as Proposition 3, assume that the cotangent vector $\left(v^{*}, \xi^{*}\right)$ is prolonged backward across the interaction time according to (4.24), together with

$$
\begin{array}{cl}
\lim _{t \rightarrow \tau-} \xi_{\alpha}^{*}(t)=\frac{1}{\dot{x}_{\alpha}^{-}} \cdot \Phi_{t}^{*}-\sum_{j \in \mathcal{J}} \frac{\dot{y}_{j}^{+}}{\dot{x}_{\alpha}^{-}} \hat{\xi}_{j}^{*+}, & \\
\lim _{t \rightarrow \tau-} \xi_{\gamma}^{*}(t)=\lim _{t \rightarrow \tau+} \xi_{\gamma}^{*}(t) & \gamma \neq \alpha .
\end{array}
$$

Then, for every solution $(v, \xi)$ of (3.18)-(3.21), the product (4.1) remains constant in time across the interaction.

Indeed, for every generalized tangent vector $(v, \xi)$ satisfying (3.19) and (3.21) across the interaction, the conditions (4.26) imply (4.25).

\section{5 - Necessary conditions for optimality}

Consider again the optimization problem (1.4)-(1.5) for the initial-boundary value problem (1.1)-(1.3). We always assume that the system satisfies the assumptions (H1) in Section 2. Moreover, we assume that the payoff functions $L=L(x, u, w), \phi=\phi(x, u)$ and $\psi=\psi(z, u)$ in (1.4) are continuously differentiable.

Let $\hat{u}$ be an optimal solution, corresponding to the control $(\hat{w}, \hat{z})$. In order to derive a set of necessary conditions, we consider the following related problem. For a given intermediate time $\bar{t} \in[0, T]$, assume that we are given a one-parameter family of perturbed initial data $u^{\varepsilon}(\bar{t}, \cdot)$, generating the tangent vector $(v(t), \xi(t))$. For $t \in[\bar{t}, T]$, call $u^{\varepsilon}=u^{\varepsilon}(t, x)$ the corresponding solutions of (1.1) and (1.3), using always the same control functions $t \mapsto(\hat{w}(t), \hat{z}(t))$. We wish to describe the change in the payoff functional $J$ in terms of the duality product with a suitable adjoint vector $\left(v^{*}, \xi^{*}\right)$. More precisely, we seek $\left(v^{*}(\bar{t}), \xi^{*}(\bar{t})\right)$ such that

$$
\begin{aligned}
\lim _{\varepsilon \rightarrow 0+} \frac{1}{\varepsilon}\left[\int_{\bar{t}}^{T}\right. & \int_{0}^{\infty} L\left(x, u^{\varepsilon}, \hat{w}\right) d x d t+\int_{0}^{\infty} \varphi\left(x, u^{\varepsilon}(T, x)\right) d x+\int_{\bar{t}}^{T} \psi\left(u^{\varepsilon}(t, 0), z(t)\right) d t \\
& \left.\quad-\int_{\bar{t}}^{T} \int_{0}^{\infty} L(x, \hat{u}, \hat{w}) d x d t+\int_{0}^{\infty} \varphi(x, \hat{u}(T, x)) d x+\int_{\bar{t}}^{T} \psi(\hat{u}(t, 0), \hat{z}(t)) d t\right] \\
& =\int_{0}^{\infty} v^{*}(\bar{t}, x) v(\bar{t}, x) d x+\sum_{\alpha} \xi_{\alpha}^{*}(\bar{t}) \xi_{\alpha}(\bar{t}) .
\end{aligned}
$$

In the following $D_{u} \phi$ denotes a partial derivative w.r.t. the $u$ component, while

$$
\Delta \varphi\left(x_{\alpha}(T)\right) \doteq \lim _{x \rightarrow x_{\alpha}(T)+} \varphi(x, \hat{u}(T, x))-\lim _{x \rightarrow x_{\alpha}(T)-} \varphi(x, \hat{u}(T, x))
$$

denotes the jump in $\phi(T, \hat{u})$ across a point $x_{\alpha}(T)$ where $\hat{u}(T, \cdot)$ is discontinuous. 
In order that the condition (5.1) be satisfied, for every tangent vector $(v, \xi)$ we should have

$$
\begin{gathered}
\int_{0}^{\infty} v^{*}(T, x) v(T, x) d x+\sum_{\alpha} \xi_{\alpha}(T) \xi_{\alpha}(T)=\int_{0}^{\infty} D_{u} \varphi(x, \hat{u}(T, x)) v(T, x) d x+\sum_{\alpha} \Delta \varphi\left(x_{\alpha}(T)\right) \xi_{\alpha}(T) \\
\frac{d}{d t}\left[\int_{0}^{\infty} v^{*}(t, x) v(t, x) d x+\sum_{\alpha} \xi_{\alpha}^{*}(t) \xi_{\alpha}(t)\right] \\
\quad=-\int_{0}^{\infty} D_{u} L(x, \hat{u}, \hat{w}) \cdot v(t, x) d x-\sum_{\alpha} \Delta L\left(x_{\alpha}(t)\right) \cdot \xi_{\alpha}-D_{u} \psi(\hat{u}(t), \hat{z}(t)) \cdot v(t, 0) .
\end{gathered}
$$

Furthermore, the duality product (4.1) should be continuous across each time $\tau$ where two shocks interact:

$$
\Delta\left\langle\left(v^{*}, \xi^{*}\right),(v, \xi)\right\rangle(\tau)=0 .
$$

Finally, at each time $\tau$ where a jump $x_{\alpha}$ hits the boundary $x=0$, we should have

$$
\Delta\left\langle\left(v^{*}, \xi^{*}\right),(v, \xi)\right\rangle(\tau)=-\frac{\xi_{\alpha}^{-}}{\dot{x}_{\alpha}^{-}} \cdot \Delta \psi(\tau)
$$

Here and in the sequel we use the notations

$$
\begin{gathered}
\Delta\left\langle\left(v^{*}, \xi^{*}\right),(v, \xi)\right\rangle(\tau) \doteq \lim _{t \rightarrow \tau+}\left\langle\left(v^{*}(t), \xi^{*}(t)\right),(v(t), \xi(t))\right\rangle-\lim _{t \rightarrow \tau-}\left\langle\left(v^{*}(t), \xi^{*}(t)\right),(v(t), \xi(t))\right\rangle, \\
\Delta L\left(x_{\alpha}(t)\right) \doteq L\left(x_{\alpha}(t), \hat{u}\left(t, x_{\alpha}+\right), \hat{w}(t)\right)-L\left(x_{\alpha}(t), \hat{u}\left(t, x_{\alpha}-\right), \hat{w}(t)\right) \\
\Delta \psi(\tau) \doteq \psi(\hat{u}(\tau+, 0), \hat{z}(\tau))-\psi(\hat{u}(\tau-, 0), \hat{z}(\tau)) .
\end{gathered}
$$

The equation (5.5) reflects the fact that, if the jump $x_{\alpha}$ is shifted by $\varepsilon \xi_{\alpha}$, then it will hit the boundary at time $\tau^{\varepsilon} \approx \tau-\varepsilon \xi_{\alpha} / \dot{x}_{\alpha}$. In the perturbed solution $u^{\varepsilon}$, the boundary payoff will thus be increased approximately by

$$
\left(\tau-\tau^{\varepsilon}\right) \cdot \Delta \psi(\tau)=\varepsilon \frac{\xi_{\alpha}}{\dot{x}_{\alpha}} \cdot \Delta \psi(\tau)
$$

A similar analysis as in the previous section shows that the identities (5.2)-(5.5) hold for every generalized tangent vectors $(v, \xi)$ provided that the adjoint vector $\left(v^{*}, \xi^{*}\right)$ satisfies the following system of equations.

Terminal conditions. At the final time $t=T$, the adjoint vector $\left(v^{*}, \xi^{*}\right)$ satisfies

$$
\begin{gathered}
v^{*}(T, x)=D_{u} \varphi(x, \hat{u}(T, x)), \\
\xi_{\alpha}^{*}(T)=\Delta \varphi\left(x_{\alpha}(T)\right) \quad \alpha=1, \ldots, N .
\end{gathered}
$$

Backward evolution equations. Outside jumps, one has

$$
v_{t}^{*}+v_{x}^{*} A(u)=-v^{*} \cdot D_{u} g(x, u, w)-D_{u} L(x, \hat{u}, \hat{w}) .
$$


Along a jump $x=x_{\alpha}(t)$, recalling (3.24)-(3.25), we have

$$
\begin{gathered}
\dot{\xi}_{\alpha}^{*}=\Delta L\left(x_{\alpha}(t)\right)-\frac{\partial \Psi_{\alpha}}{\partial \xi_{\alpha}} \cdot \xi_{\alpha}^{*}-\sum_{j^{ \pm} \in \mathcal{O}}\left|\lambda_{j}^{ \pm}-\dot{x}_{\alpha}\right| \frac{\partial V_{\alpha}^{j}}{\partial \xi_{\alpha}} \cdot v_{j \pm}^{*}, \\
v_{i \pm}^{*}=\frac{1}{\left|\lambda_{i}^{ \pm}-\dot{x}_{\alpha}\right|}\left\{\frac{\partial \Psi_{\alpha}}{\partial v_{i \pm}} \cdot \xi_{\alpha}^{*}+\sum_{j^{ \pm} \in \mathcal{O}}\left|\lambda_{j}^{ \pm}-\dot{x}_{\alpha}\right| \frac{\partial V_{\alpha}^{j}}{\partial v_{i \pm}} \cdot v_{j \pm}^{*}\right\} \quad i^{ \pm} \in \mathcal{I},
\end{gathered}
$$

Moreover, along the boundary $x=0$ one has

$$
v_{i}^{*}=\sum_{j=p+1}^{n}\left|\frac{\lambda_{j}}{\lambda_{i}}\right| \frac{\partial \widetilde{V}^{j}}{\partial v_{i}} \cdot v_{j}^{*}+\frac{1}{\left|\lambda_{i}\right|} D_{u} \psi \cdot\left(r_{i}+\sum_{j=p+1}^{n} \frac{\partial \widetilde{V}^{j}}{\partial v_{i}} r_{j}\right) \quad i=1, \ldots, p,
$$

where all functions are evaluated at $x=0, u=\hat{u}(t), z=\hat{z}(t)$. We recall that the functions $\widetilde{V}^{j}$, providing the size of the outgoing components at the boundary in terms of the incoming components, were introduced in (2.17). The motivation for the last term in (5.11) is illustrated in fig. 3. Assume that we increase the $i$-th incoming component of $\hat{u}$ in a neighborhood of $x=0$. More precisely, for a fixed $i \in\{1, \ldots, p\}$, let us replace $\hat{u}(t, x)$ with $u^{\varepsilon}(t, x)=\hat{u}(t, x)+\varepsilon r_{i}$ for $x \in[0, h]$. Then, on a time interval $I \approx\left[t, t-h / \lambda_{i}\right]$, the boundary values of $u$ will change according to

$$
u^{\varepsilon}(\tau, 0)=\hat{u}(\tau, 0)+\varepsilon\left(r_{i}+\sum_{j>p} \frac{\partial \widetilde{V}^{j}}{\partial v_{i}} r_{j}\right) .
$$

In turn, the change in the time-integral of $\psi$ along the boundary is approximately given by

$$
\int_{t}^{t-h / \lambda_{i}} D_{u} \psi \cdot \varepsilon\left(r_{i}+\sum_{j>p} \frac{\partial \widetilde{V}^{j}}{\partial v_{i}} r_{j}\right) d x
$$

Restarting conditions across interactions. At a time $\tau$ where two jumps interact, because of (5.4) the restarting conditions for the adjoint vector $\left(v^{*}, \xi^{*}\right)$ are still provided by $(4.16)$ and (4.18)-(4.20).

At a time $\tau$ where the jump $x_{\alpha}$ hits the boundary, because of (5.5) the restarting conditions are provided by (4.24), (4.27), while (4.26) must be replaced by

$$
\lim _{t \rightarrow \tau-} \xi_{\alpha}^{*}(t)=\frac{1}{\dot{x}_{\alpha}^{-}} \cdot \Phi_{t}^{*}-\sum_{j \in \mathcal{J}} \frac{\dot{y}_{j}^{+}}{\dot{x}_{\alpha}^{-}} \hat{\xi}_{j}^{*+}+\frac{1}{\dot{x}_{\alpha}^{-}} \Delta \psi(\tau) .
$$

Together, these conditions allow us to construct the cotangent vectors $\left(v^{*}, \xi^{*}\right)$ backward in time, also across interactions.

We can now discuss a necessary condition for optimality of a solution to the problem (1.1)(1.5). By the results in [BM1], the change in $\hat{u}(T, \cdot)$ can be described up to first order in terms of a generalized tangent vector, provided that all solutions $u^{\varepsilon}$ remain piecewise Lipschitz continuous, 


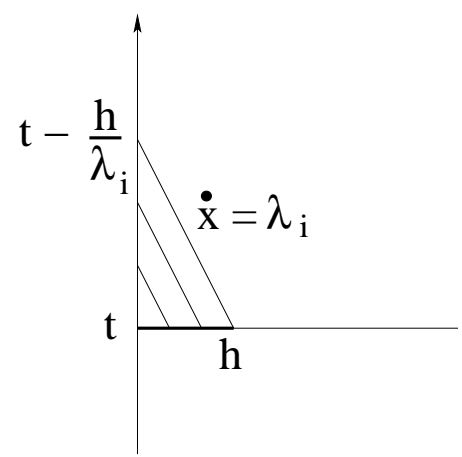

figure 3

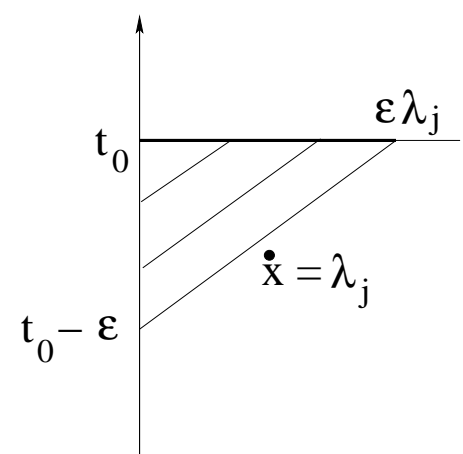

figure 4

with the same number of discontinuities as $\hat{u}$. To ensure this condition, we need some stronger regularity assumption on the reference solution $\hat{u}$. Namely

(H2) The function $\hat{u}=\hat{u}(t, x)$ is piecewise $\mathcal{C}^{1}$ on $[0, T] \times \mathbb{R}$, with finitely many jumps and finitely many lines of weak discontinuities (where $\hat{u}$ is continuous but its derivative $\hat{u}_{x}$ is discontinuous). These curves where $\hat{u}$ is not smooth can interact only two at a time. Similarly, they can touch the boundary only one at at a time.

The above assumption states that all curves of singularities in the reference solution $\hat{u}$ are in "generic position".

Theorem 1 (Necessary conditions for optimality). Let the system (1.1) satisfy the assumptions (H1) and assume that the control functions $t \mapsto(\hat{w}(t), \hat{z}(t)) \in W \times Z \subseteq \mathbb{R}^{m} \times \mathbb{R}^{m^{\prime}}$ are continuously differentiable and provide an optimal solution to the maximization problem (1.1)(1.5). Moreover, assume that the optimal trajectory satisfies the regularity conditions (H2).

Define the adjoint vector $\left(v^{*}, \xi^{*}\right)$ as the solution to the backward linear evolution problem (5.6)-(5.11), together with the restarting conditions (4.16), (4.18)-(4.20) at times of interactions between two jumps, and the restarting conditions (4.24), (4.27) and (5.12) at times where a jump hits the boundary.

Then, for almost every time $t \in[0, T]$ and every $w \in W, z \in Z$ one has

$$
\begin{gathered}
\int_{0}^{\infty}\left[v^{*}(t, x) \cdot D_{w} g(x, \hat{u}(t, x), \hat{w}(t))+D_{w} L(x, \hat{u}(t, x), \hat{w}(t))\right] d x \cdot(w-\hat{w}(t)) \leq 0, \\
\left(D_{z} \psi(\hat{u}(t, 0), \hat{z}(t))+\sum_{j=p+1}^{n} v_{j}^{*}(t, 0) \lambda_{j}(0, t) \Lambda_{j}(t)\right) \cdot(z-\hat{z}(t)) \leq 0 .
\end{gathered}
$$

Here $\Lambda_{j}(t)=\Lambda_{j}(u(0, t), z(t))$ is the linear map defined at (2.19).

Proof. Let $\varphi: \mathbb{R} \mapsto[0,2]$ be a smooth function with compact support, such that

$$
\int_{-1}^{0} \varphi(s) d s=1, \quad \varphi(s)=0 \quad \text { if } \quad s \notin[-1,0] .
$$

Fix a time $t_{0}>0$ and a vector $w \in W \subset \mathbb{R}^{m}$, and consider the perturbed control function

$$
w^{\varepsilon}(t) \doteq \hat{w}(t)+\varepsilon^{2} \varphi\left(\frac{t-t_{0}}{\varepsilon}\right) \cdot(w-\hat{w}(t)) .
$$


Let $u^{\varepsilon}=u\left(w^{\varepsilon}, \hat{z}\right)$ be the corresponding solution of (1.1)-(1.3). Notice that here we are changing the control function $\hat{w}$ but not the boundary control $\hat{z}$. As $\varepsilon \rightarrow 0+$, the control $w^{\varepsilon}$ converges to $\hat{w}$ in $\mathcal{C}^{1}$. Therefore, the perturbed solutions $u^{\varepsilon}$ have the same structure as the reference solution $\hat{u}$. We now compute

$$
\begin{aligned}
\lim _{\varepsilon \rightarrow 0+} & \frac{J\left(u^{\varepsilon}, w^{\varepsilon}, \hat{z}\right)-J(\hat{u}, \hat{w}, \hat{z})}{\varepsilon^{3}} \\
= & \lim _{\varepsilon \rightarrow 0+} \frac{1}{\varepsilon^{3}} \int_{t_{0}-\varepsilon}^{t_{0}} \int_{0}^{\infty} D_{w} L(x, \hat{u}(t, x), \hat{w}(t)) \cdot \varepsilon^{2} \varphi\left(\frac{t-t_{0}}{\varepsilon}\right) \cdot(w-\hat{w}(t)) d x d t \\
& \quad+\lim _{\varepsilon \rightarrow 0+} \frac{1}{\varepsilon^{3}} \int_{0}^{\infty} v^{*}\left(t_{0}, x\right)\left[u^{\varepsilon}\left(t_{0}, x\right)-\hat{u}\left(t_{0}, x\right)\right] d x \\
= & \left.\int_{0}^{\infty} D_{w} L\left(x, \hat{u}\left(t_{0}, x\right), \hat{w}\left(t_{0}\right)\right)\right] d x \cdot\left(w-\hat{w}\left(t_{0}\right)\right) d x \\
& \quad+\int_{0}^{\infty}\left[v^{*}\left(t_{0}, x\right) \cdot D_{w} g\left(x, \hat{u}\left(t_{0}, x\right), \hat{w}\left(t_{0}\right)\right) d x\right.
\end{aligned}
$$

The optimality assumption implies that the left hand side of $(5.15)$ is $\leq 0$. This yields (5.13), at time $t=t_{0}$. Notice that the displacement of the jump points in the perturbed solution $u^{\varepsilon}$ is a higher order infinitesimal, as $\varepsilon \rightarrow 0$. Namely

$$
x_{\alpha}^{\varepsilon}\left(t_{0}\right)-x_{\alpha}\left(t_{0}\right)=\mathcal{O}\left(\varepsilon^{4}\right) .
$$

For this reason, the terms $\xi_{\alpha}^{*}\left(t_{0}\right) \cdot\left[x_{\alpha}^{\varepsilon}\left(t_{0}\right)-x_{\alpha}\left(t_{0}\right)\right]$ do not appear on the right hand side of (5.15).

Next, fix a time $t_{0}>0$ and a vector $z \in Z \subset \mathbb{R}^{m^{\prime}}$, and consider the perturbed control function

$$
z^{\varepsilon}(t) \doteq \hat{z}(t)+\varepsilon^{2} \varphi\left(\frac{t-t_{0}}{\varepsilon}\right) \cdot(z-\hat{z}(t))
$$

Let $u^{\varepsilon}=u\left(\hat{w}, z^{\varepsilon}\right)$ be the corresponding solution of (1.1)-(1.3). Notice that now we are only changing the boundary control $\hat{z}$ in a backward neighborhood of the time $t_{0}$. As $\varepsilon \rightarrow 0+$, the control $z^{\varepsilon}$ converges to $\hat{z}$ in $\mathcal{C}^{1}$. Therefore, the perturbed solutions $u^{\varepsilon}$ have again the same structure as the reference solution $\hat{u}$. We now compute

$$
\begin{aligned}
\lim _{\varepsilon \rightarrow 0+} & \frac{J\left(u^{\varepsilon}, \hat{w}, z^{\varepsilon}\right)-J(\hat{u}, \hat{w}, \hat{z})}{\varepsilon^{3}} \\
= & \lim _{\varepsilon \rightarrow 0+} \frac{1}{\varepsilon^{3}} \int_{t_{0}-\varepsilon}^{t_{0}} D_{z} \psi(\hat{u}(t, 0), \hat{z}(t),) \cdot \varepsilon^{2} \varphi\left(\frac{t-t_{0}}{\varepsilon}\right) \cdot(z-\hat{z}(t)) d x d t \\
& \quad+\lim _{\varepsilon \rightarrow 0+} \frac{1}{\varepsilon^{3}} \int_{0}^{\infty} v^{*}\left(t_{0}, x\right)\left[u^{\varepsilon}\left(t_{0}, x\right)-\hat{u}\left(t_{0}, x\right)\right] d x \\
= & D_{z} \psi\left(\hat{u}\left(t_{0}, 0\right), \hat{z}\left(t_{0}\right),\right) \cdot\left(z-\hat{z}\left(t_{0}\right)\right) \\
& \quad+\sum_{j=p+1}^{n} v_{j}^{*}\left(t_{0}, 0\right) \lambda_{j}\left(\hat{u}\left(0, t_{0}\right)\right) \cdot \frac{\partial \widetilde{V}^{j}}{\partial z}\left(t_{0}\right) \cdot\left(z-\hat{z}\left(t_{0}\right)\right) .
\end{aligned}
$$

By the optimality assumption, the left hand side of (5.16) must be $\leq 0$. This yields (5.14), at time $t=t_{0}$. 
The derivation of the last term in (5.16) is illustrated in fig. 4. Assume that the boundary control is changed from $\hat{z}$ to $\hat{z}+\varepsilon^{2}(z-\hat{z})$ on the time interval $\left[t_{0}-\varepsilon, t_{0}\right]$. Then the outgoing components are increased in the amounts

$$
\left\langle l_{i}(\hat{u}), u^{\varepsilon}(t, 0)-\hat{u}(t, 0)\right\rangle \approx \frac{\partial \widetilde{V}^{j}}{\partial z} \cdot \varepsilon^{2}(z-\hat{z}(t)) \quad j=p+1, \ldots, n .
$$

These components move away from the boundary at characteristic speeds $\lambda_{j}$ At time $t_{0}$, we thus have

$$
u^{\varepsilon} \approx \hat{u}+\sum_{j=n-p+1}^{n} \chi_{\left[0, \varepsilon \lambda_{j}\right]} \cdot \frac{\partial \widetilde{V}^{j}}{\partial z} \cdot \varepsilon^{2}(z-\hat{z}) r_{j} .
$$

Integrating w.r.t. $x$, one thus obtains the additional factors $\lambda_{j}$ on the right hand side of (5.16).

\section{6 - Concluding remarks}

The aim of this section is to clarify a limitation of the present analysis, and outline possible research directions.

Remark 4. The linearized system of equations describing the evolution of a generalized tangent vector $(v, \xi) \in \mathbf{L}^{1} \times \mathbb{R}^{N}$ is meaningful as long as the reference solution $u$ remains piecewise Lipschitz continuous. The same is true for the optimality conditions (5.13)-(5.14). However, in order to prove that these conditions are necessary for optimality, we needed to assume that the reference solution is $C^{1}$ outside the shock curves.

This technical point can be explained as follows. The evolution equations for a generalized tangent vectors were derived in [BM1] assuming that we have a one-parameter family of perturbed solutions $u^{\theta}$, and that each of these solutions has exactly the same structure (i.e. the same number of shock curves) as the reference solution $u$.

To use this result, when we derive necessary conditions for the optimality of a solution $u$ to (1.1)-(1.3) we need to construct small perturbations $u^{\theta}$ having the same shock structure as $u$. This is easy to do if $u$ is $\mathcal{C}^{1}$ outside shock curves, but may be not possible in the piecewise Lipschitz continuous case.
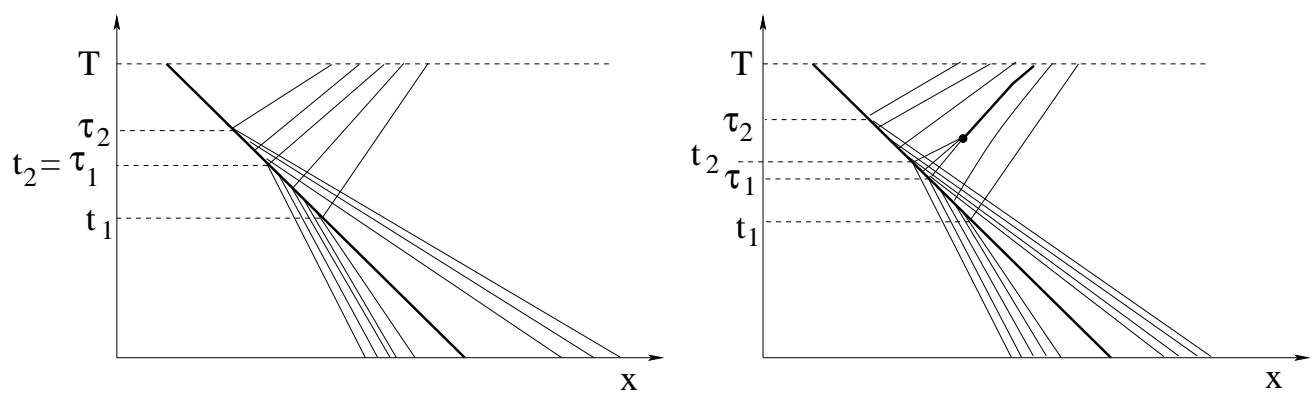

figure 5

For example, consider the $2 \times 2$ system of isentropic gas dynamics:

$$
v_{t}+u_{x}=0, \quad u_{t}+p(v)_{x}=0 .
$$


Consider a solution which initially consists of two rarefaction waves of the first family approaching a shock of the first family (fig. 5). When the interactions take place, it is well known that a compression wave of the second family is generated. Assume that the two waves impinge on the shock during time intervals $\left[t_{1}, t_{2}\right]$ and $\left[\tau_{1}, \tau_{2}\right]$ respectively, with $t_{2}=\tau_{1}$. One can arrange things so that the outgoing compression 2 -waves have a sufficiently small gradient and do not break throughout the time interval $\left[t_{1}, T\right]$. Next, consider a small perturbation $u^{\theta}$. It is quite possible that the time intervals $\left[t_{1}^{\theta}, t_{2}^{\theta}\right]$ and $\left[\tau_{1}^{\theta}, \tau_{2}^{\theta}\right]$ now overlap, so that $t_{2}^{\theta}>\tau_{1}^{\theta}$. The outgoing compression 2 -wave leaving the shock during the time subinterval $\left[\tau_{1}^{\theta}, t_{2}^{\theta}\right]$ will have a much steeper gradient. In particular, it can break producing a 2 -shock much before time $T$. This example shows that, for piecewise Lipschitz continuous solutions, an arbitrarily small perturbation can have an entirely different shock structure.

It seems reasonable to conjecture that the optimality conditions (5.13)-(5.14) remain valid also for piecewise Lipschitz solutions. However, a rigorous proof will likely need to consider more general perturbations, possibly with different shock structures. In this direction, we recall that an extension of the concept of "shift differential", as introduced in [BG, BL], encounters serious difficulties when applied to systems. Indeed, as shown by the counterexample in [Bi1], shift differentials may not be well defined along the flow of a $2 \times 2$ hyperbolic system.

Remark 5. In the above example, the solution is still piecewise smooth. However, the solution is continuous but not differentiable along the characteristic curves (of the first family) starting at the points $a, b, c$, and $d$. The two curves starting from $b$ and $c$ hit the shock exactly at the same time $t=t_{2}=\tau_{1}$. This situation is not covered by our theorem, because the double interaction is structurally unstable, and the regularity assumptions (H2) are not satisfied.

Remark 6. If one is interested in the numerical solution of an optimization problem, the use of necessary conditions may not be the most efficient approach. In particular, if the objective function is not differentiable but only Lipschitz continuous, more robust methods can be desirable. To solve the problem (1.1)-(1.5), a natural strategy is to consider a family of approximations to the system of balance laws (1.1) and compute an optimal solution within these approximations. Several approximations methods have been considered in the literature: the Glimm scheme [G, $\mathrm{S}]$, front tracking $[\mathrm{B}, \mathrm{HR}]$, semi-discrete and fully discrete schemes [LV], vanishing viscosity [BiB], relaxation approximations [JX]. Rigorous convergence results and error estimates are known [Bi2, $\mathrm{Bi} 3, \mathrm{BM} 3, \mathrm{BY}]$.

In general, the nonlinear optimization problem (1.1)-(1.5) will have more than one local minima. The global minimum can be searched by carrying out several local minimization procedures on families of approximate solutions. A key ingredient for this strategy to work is that the approximation schemes do not introduce new point of (spurious) local minima. It is reasonable to expect this is the case for vanishing viscosity approximations, but the situation is far from clear in connection with approximations, such as front tracking or the Glimm scheme, which may not depend continuously on the initial data.

Acknowledgements. The work of the first author has been supported by the N.S.F. Grant DMS-0505430 - Applied Mathematics: Hyperbolic systems of conservation laws.

The authors wish to thank Fabio Ancona for carefully reading the first version of the paper, and for several useful suggestions. 


\section{REFERENCES}

[AC] D. Amadori and R. M. Colombo Continuous Dependence for 2x2 Conservation Laws with Boundary J. Differential Equations 138 (1997), 229-266.

[Bi1] S. Bianchini, On the shift differentiability of the flow generated by a hyperbolic system of conservation laws, Discr. Cont. Dynam. Syst. 6 (2000), 329-350.

[Bi2] S. Bianchini, BV solutions of the semidiscrete upwind scheme. Arch. Rational Mech. Anal. 167 (2003), 1-81.

[Bi3] S. Bianchini, Hyperbolic limit of the Jin-Xin relaxation model, Comm. Pure Appl. Math., to appear.

[BiB] S. Bianchini and A. Bressan, Vanishing viscosity solutions of nonlinear hyperbolic systems, Annals of Mathematics 161 (2005), 223-342.

[BJ] F. Bouchut and F. James, Differentiability w.r.t. the initial data for scalar conservation laws, in Hyperbolic Problems, Theory, Numerics, Applications, Proc. of the Conference in Zürich (1998), 113-118.

[B] A. Bressan, Hyperbolic Systems of Conservation Laws. The One Dimensional Cauchy Problem, Oxford University Press, 2000.

[BG] A. Bressan and G. Guerra, Shift differentiability of the flow generated by a conservation law, Discr. Cont. Dynam. Syst. 3 (1997), 35-58.

[BL] A. Bressan and M. Lewicka, Shift differentials of maps in BV spaces, in "Nonlinear Theory of Generalized Functions", M. Grosser, G. Hörmann, M. Kunziger and M. Oberguggenberger Eds., Chapman \& Hall/CRC Research Notes in Mathematics (1999), pp.47-61

[BM1] A. Bressan and A. Marson A variational calculus for discontinuous solutions of conservative systems, Comm. Part. Diff. Equat. 20 (1995), 1491-1552.

[BM2] A. Bressan and A. Marson A maximum principle for optimally controlled systems of conservation laws, Rend. Sem. Mat. Univ. Padova 94 (1995), 79-94

[BM3] A. Bressan and A. Marson Error bounds for a deterministic version of the Glimm scheme, Arch. Rational Mech. Anal. 142 (1998), 155-176.

[BY] A. Bressan and T. Yang, On the rate of convergence of vanishing viscosity approximations, Comm. Pure Appl. Math 57 (2004), 1075-1109

[D] C. Dafermos, Hyperbolic Conservation Laws in Continuum Physics, Springer-Verlag, Berlin 2000 .

[G] J. Glimm, Solutions in the large for nonlinear hyperbolic systems of equations, Comm. Pure Appl. Math. 18 (1965), 697-715.

[HR] H. Holden and N. H. Risebro Front Tracking for Hyperbolic Conservation Laws, Springer Verlag, New York 2002. 
[JX] S. Jin and Z. Xin, The relaxation schemes for systems of conservation laws in arbitrary space dimensions, Comm. Pure Appl. Math. 48 (1955), 235-277.

[LV] R. J. LeVeque, Numerical Methods for Conservation Laws, Second Edition, Birkhäuser 1992.

[Li] Ta Tsien Li, Global Classical Solutions for Quasilinear Hyperbolic Systems. John Wiley \& Sons, Ltd., Chichester, 1994.

[RY] B. L. Rozdesvenskii and N. Yanenko, Systems of Quasilinear Equations and Their Applications to Gas Dynamics. A.M.S. Translations of Mathematical Monographs 55, Providence, 1983.

[S] J. Smoller, "Shock waves and reaction-diffusion equations", Springer-Verlag, New York, 1983. 